\title{
Buchbesprechungen - Book Reviews - Livres Nouveaux
}

Caroli, J.: Les ictères par retention. Masson \& Cie., Paris 1956. 481 p.

Tous ceux qui se sont trouvés un jour devant le difficile problème diagnostique d'un ictère, seront heureux d'apprendre que Caroli vient de réunir dans un remar-quable volume les principes et les idées qu'il fallait jusqu'ici chercher dans de nombreux articles écrits de sa plume.

L'intention de Tauteur a été de nous offrir un manuel pratique de diagnostic des ictères par retention et il a, dans ce but, établi des regies simples et fidèles, souvent énoncées sous forme de lois ou d'aphorismes. En effet, dans environ $70 \%$ des cas, la sémiologie bien codifiée offre un diagnostic de certitude; 2 ou 3 fois sur 10, cependant, divereses investigations sont nécessaires et permettent, bien conduites, d'éviter toute erreur de diagnostic.

Ce livre d'une clarté toute cartésienne et d'une langue parfaite se lit avec un plaisir et un intérêt constants, grace aussi à un excellent choix de la typographie, mettant en evidence les faits importants. Les aphorismes, que $\Gamma$ auteur a voulus un peu schématiques, devraient dorénavant constituer $\Gamma \mathrm{ABC}$ de tout etudiant en médecine et de tout médecin en face du problème de Tictère. Caroli nous offre là un «clavier» de symptômes et il suffit d'appuyer sur les «touches»» correspondantes pour qu'aussitôt $« \Gamma$ accord $»$ realise donne le diagnostic.

L'ouvrage est construit dans $\Gamma$ ordre même où $\Gamma$ on doit procéder avec un malade. C'est tout d'abord Yétape clínique qui comprend la recherche des signes fonction-nels (prodromes surtout) et des signes physiques, les regies tirées de $\Gamma$ anamnèse, de certaines associations et de revolution.

Puis vient Yétape biologique; Tauteur s'en tient à juste titre à une batterie de tests assez simples mais fidèles et dont le groupement suffit pour apporter tous les renseignements qu'on peut attendre du laboratoire. II s'agit des tests de McLagan et Hanger, du cholesterol total, de la galactosurie provoquée, éventuellement de Гélectrophorèse. L'hémogramme donne d'utiles indications. Le tubage duodenal, surtout lorsqu'il est combine avec le temps d'apparition de la B.S.P. (épreuve de

Buchbesprechungen - Book Reviews - Livres Nouveaux 63

Caroli) constitue une épreuve précieuse pour le diagnostic différentiel des ictères incomplete. Uétape radiologique préopératoíre comprend la radiologie simple de l'hypocondre droit, la duodénographie barytée, la cholécystographie orale, éventuellement $\Gamma$ an-giocholégraphie i.v. et parfois la splénoportographie.

Plus de la moitié du volume est encore consacrée à des examens qui concernent davantage le spécialiste, à savoir la laparoscopie (avec ou sans angiocholégraphie selon Royer), la ponctíonbiopsíe hépatique et la radiomanométríe opératoire sous contrôle radiologique permanent. Ce magnifique ouvrage est illustré de 308 figures et de 2 planches hors texte en couleurs. La plupart des radiographies sont empruntées à Porcher et $\Gamma$ édition a été confiée à Masson; ce sont là deux garanties de perfection technique.

Le livre de Caroli vient combler une lacune et il nous semble répondre exacte-ment à ce que Гon pouvait attendre d'un tel maître sur un tel sujet. II devrait trou-ver sa place dans la bibliothèque - 
ou mieux, sur la table de travail - des spécia-listes aussi bien que des internistes, des chirurgiens et des étudiants.

B. Wíssmer, Geneve

Morítsch, P.: Schmerzverhütung bei chirurgischen Eingriffen. 3. neubearbeitete Aufl. Verlag Wilhelm Maudrich, Wien 1956. VIII + 364 S., 104 Abb. sFr. 50.-.

Nachdem sich die Anästhesie in schneller Entwicklung zum eigenen Fach aus-gebaut hat, drängen sich auch entsprechende entwickelte Lehr- und Nachschlage-werke auf. Wer sich in zusammenfassender Weise über die Möglichkeiten der Schmerzausschaltung orientieren will, kann das Buch zur Hand nehmen, wenn auch ohne die Erwartung vollständige Angaben zu finden.

Auf 364 Seiten beschreibt Morítsch, oft von den historischen Methoden aus-gehend, Entwicklung und Technik der allgemeinen Narkose und der Lokal- und Leitungsanästhesie. Prämedikation, absichtliche Blutdrucksenkung, Relaxations-methoden, Narkosetheorie, Unterkühlungs-, Hibernations- und Intubationstechnik werden dargelegt, bei ihrer heutigen Bedeutung jedoch in viel zu knapper Fassung. Dagegen finden wir eine ausführliche und ausgezeichnete Beschreibung der Lokal-anästhesie und ihrer Pharmaka. Den Besonderheiten bei alten Patienten und Dia-betikern ist ein eigenes Kapitel gewidmet. Eine Bereicherung erfuhr die dritte Auf-lage durch Erwähnung der transthorakalen Herzmassage bei Herzstillstand.

So erfreulich die Zusammenfassung der lokalen und allgemeinen Anästhesie in einem einzigen Buch für den Chirurgen ist, kann dieses nicht unbedingt als Berater empfohlen werden. Außer einigen irreführenden Druckfehlern ist vor ahem die Bebilderung zu kritisieren. Sie ist den Fortschritten der Anästhesie nicht angepaßt worden. Man wünscht sich an Stelle alter und unwichtiger Bilder (z. B. Abbildungen 5-10 usw.) oder geradezu verkehrter Instruktionen (z. B. Abbildungen 19-21) Bilder, die das Wesentliche und allgemein anerkannte Lehren illustrieren. Bei der intravenösen Narkose ist z. B. den Weckmitteln viel Raum gegeben worden, jedoch die Wegleitung, wie gefährliche Situationen zu vermeiden sind, ist zu kurz weggekommen. Zusammenfassend muß man feststellen, daß das Buch bei einem vorzüglichen Teil über Lokalanästhesie einen Narkoseteil aufweist, der mindestens stellenweise rückständig geblieben ist. P. Ganz, Basel 